\title{
Search for Lorentz and $C P T$ violation using sidereal time dependence of neutrino flavor transitions over a short baseline
}

K. Abe,${ }^{47}$ J. Amey,${ }^{16}$ C. Andreopoulos,${ }^{45,26}$ M. Antonova, ${ }^{21}$ S. Aoki, ${ }^{23}$ A. Ariga, ${ }^{1}$ S. Assylbekov, ${ }^{7}$ D. Autiero, ${ }^{28}$ S. Ban, ${ }^{24}$ F. C. T. Barbato, ${ }^{18}$ M. Barbi ${ }^{39}$ G. J. Barker ${ }^{55}$ G. Barr, ${ }^{35}$ C. Barry ${ }^{26}$ P. Bartet-Friburg, ${ }^{36}$ M. Batkiewicz, ${ }^{12}$ V. Berardi, ${ }^{17}$ S. Berkman, ${ }^{3,51}$ S. Bhadra ${ }^{60}$ S. Bienstock, ${ }^{36}$ A. Blondel, ${ }^{11}$ S. Bolognesi,${ }^{5}$ S. Bordoni $,{ }^{14},{ }^{\dagger}$ S. B. Boyd ${ }^{55}$ D. Brailsford, ${ }^{25}$ A. Bravar, ${ }^{11}$ C. Bronner, ${ }^{22}$ M. Buizza Avanzini, ${ }^{9}$ R. G. Calland ${ }^{22}$ T. Campbell, ${ }^{7}$ S. Cao, ${ }^{13}$ S. L. Cartwright, ${ }^{43}$ R. Castillo, ${ }^{14}$ M. G. Catanesi, ${ }^{17}$ A. Cervera, ${ }^{15}$ A. Chappell,${ }^{55}$ C. Checchia,${ }^{19}$ D. Cherdack, ${ }^{7}$ N. Chikuma, ${ }^{46}$ G. Christodoulou, ${ }^{26}$ A. Clifton, ${ }^{7}$ J. Coleman, ${ }^{26}$ G. Collazuol, ${ }^{19}$ D. Coplowe, ${ }^{35}$ L. Cremonesi, ${ }^{38}$ A. Cudd, ${ }^{29}$ A. Dabrowska, ${ }^{12}$ G. De Rosa, ${ }^{18}$ T. Dealtry, ${ }^{25}$ P. F. Denner, ${ }^{55}$ S. R. Dennis ${ }^{26}$ C. Densham, ${ }^{45}$ D. Dewhurst,${ }^{35}$ F. Di Lodovico, ${ }^{38}$ S. Di Luise, ${ }^{10}$ S. Dolan, ${ }^{35}$ O. Drapier, ${ }^{9}$ K. E. Duffy, ${ }^{35}$ J. Dumarchez, ${ }^{36}$ M. Dunkman, ${ }^{29}$ M. Dziewiecki, ${ }^{54}$ S. Emery-Schrenk, ${ }^{5}$ A. Ereditato, ${ }^{1}$

T. Feusels, ${ }^{3,51}$ A. J. Finch ${ }^{25}$ G. A. Fiorentini, ${ }^{60}$ M. Friend, ${ }^{13,}$ Y. Fujiii, ${ }^{13, *}$ D. Fukuda,${ }^{33}$ Y. Fukuda, ${ }^{30}$ A. P. Furmanski, ${ }^{55}$ V. Galymov, ${ }^{28}$ A. Garcia, ${ }^{14}$ S. G. Giffin, ${ }^{39}$ C. Giganti, ${ }^{36}$ F. Gizzarelli, ${ }^{5}$ T. Golan, ${ }^{58}$ M. Gonin, ${ }^{9}$ N. Grant, ${ }^{55}$ D. R. Hadley, ${ }^{55}$ L. Haegel, ${ }^{11}$ J. T. Haigh, ${ }^{55}$ P. Hamilton, ${ }^{16}$ D. Hansen, ${ }^{37}$ J. Harada, ${ }^{34}$ T. Hara, ${ }^{23}$ M. Hartz, ${ }^{22,51}$ T. Hasegawa, ${ }^{13, \sharp}$

N. C. Hastings, ${ }^{39}$ T. Hayashino, ${ }^{24}$ Y. Hayato, ${ }^{47,22}$ R. L. Helmer, ${ }^{51}$ M. Hierholzer, ${ }^{1}$ A. Hillairet, ${ }^{52}$ A. Himmel, ${ }^{8}$ T. Hiraki, ${ }^{24}$ A. Hiramoto, ${ }^{24}$ S. Hirota, ${ }^{24}$ M. Hogan, ${ }^{7}$ J. Holeczek, ${ }^{44}$ F. Hosomi, ${ }^{46}$ K. Huang, ${ }^{24}$ A. K. Ichikawa, ${ }^{24}$ K. Ieki, ${ }^{24}$ M. Ikeda, ${ }^{47}$ J. Imber, ${ }^{9}$ J. Insler, ${ }^{27}$ R. A. Intonti, ${ }^{17}$ T. J. Irvine, ${ }^{48}$ T. Ishida, ${ }^{13, \$}$ T. Ishii, ${ }^{13, *}$ E. Iwai, ${ }^{13}$ K. Iwamoto, ${ }^{40}$ A. Izmaylov ${ }^{15,21}$ A. Jacob, ${ }^{35}$ B. Jamieson, ${ }^{57}$ M. Jiang, ${ }^{24}$ S. Johnson, ${ }^{6}$ J. H. Jo,${ }^{32}$ P. Jonsson, ${ }^{16}$ C. K. Jung, ${ }^{32,8}$ M. Kabirnezhad ${ }^{31}$ A. C. Kaboth, ${ }^{41,45}$ T. Kajita, ${ }^{48,8}$ H. Kakuno, ${ }^{49}$ J. Kameda ${ }^{47}$ D. Karlen ${ }^{52,51}$ I. Karpikov, ${ }^{21}$ T. Katori, ${ }^{38}$ E. Kearns, ${ }^{2,22,8}$ M. Khabibullin, ${ }^{21}$ A. Khotjantsev, ${ }^{21}$ D. Kielczewska, ${ }^{53,{ }^{*}}$ T. Kikawa, ${ }^{24}$ H. Kim, ${ }^{34}$ J. Kim, ${ }^{3,51}$ S. King, ${ }^{38}$ J. Kisiel, ${ }^{44}$ A. Knight, ${ }^{55}$ A. Knox,${ }^{25}$ T. Kobayashi, ${ }^{13, *}$ L. Koch, ${ }^{42}$ T. Koga, ${ }^{46}$ A. Konaka, ${ }^{51}$ K. Kondo, ${ }^{24}$ A. Kopylov, ${ }^{21}$ L. L. Kormos, ${ }^{25}$ A. Korzenev, ${ }^{11}$ Y. Koshio, ${ }^{33, \|}$ K. Kowalik, ${ }^{31}$ W. Kropp, ${ }^{4}$ Y. Kudenko ${ }^{21,9}$ R. Kurjata, ${ }^{54}$ T. Kutter, ${ }^{27}$ J. Lagoda,${ }^{31}$ I. Lamont, ${ }^{25}$ M. Lamoureux, ${ }^{5}$ E. Larkin, ${ }^{55}$ P. Lasorak, ${ }^{38}$ M. Laveder, ${ }^{19}$ M. Lawe ${ }^{25}$ M. Lazos, ${ }^{26}$ M. Licciardi, ${ }^{9}$ T. Lindner ${ }^{51}$ Z. J. Liptak, ${ }^{6}$ R. P. Litchfield, ${ }^{16}$ X. Li, ${ }^{32}$ A. Longhin, ${ }^{19}$ J. P. Lopez,${ }^{6}$ T. Lou, ${ }^{46}$ L. Ludovici, ${ }^{20}$ X. Lu, ${ }^{35}$ L. Magaletti, ${ }^{17}$ K. Mahn, ${ }^{29}$ M. Malek, ${ }^{43}$ S. Manly, ${ }^{40}$ L. Maret, ${ }^{11}$ A. D. Marino, ${ }^{6}$ J. Marteau, ${ }^{28}$ J. F. Martin, ${ }^{50}$ P. Martins, ${ }^{38}$ S. Martynenko, ${ }^{32}$ T. Maruyama, ${ }^{13,5}$ V. Matveev, ${ }^{21}$ K. Mavrokoridis, ${ }^{26}$ W. Y. Ma ${ }^{16}$ E. Mazzucato, ${ }^{5}$ M. McCarthy, ${ }^{60}$ N. McCauley, ${ }^{26}$ K. S. McFarland, ${ }^{40}$ C. McGrew, ${ }^{32}$ A. Mefodiev, ${ }^{21}$ C. Metelko, ${ }^{26}$ M. Mezzetto, ${ }^{19}$ P. Mijakowski, ${ }^{31}$ A. Minamino, ${ }^{59}$ O. Mineev, ${ }^{21}$ S. Mine, ${ }^{4}$ A. Missert, ${ }^{6}$ M. Miura, ${ }^{47, \|}$ S. Moriyama, ${ }^{47, \|}$ J. Morrison, ${ }^{29}$ Th. A. Mueller, ${ }^{9}$ S. Murphy, ${ }^{10}$ J. Myslik, ${ }^{52}$ T. Nakadaira, ${ }^{13, \$}$ M. Nakahata, ${ }^{47,22}$ K. G. Nakamura ${ }^{24}$ K. Nakamura, ${ }^{22,13, \$}$ K. D. Nakamura, ${ }^{24}{ }^{24}$ Y. Nakanishi, ${ }^{24}$ S. Nakayama, ${ }^{47, \|}$ T. Nakaya ${ }^{24,22}$ K. Nakayoshi, ${ }^{13, \$}$ C. Nantais,${ }^{50}$ C. Nielsen,${ }^{3}$ M. Nirkko, ${ }^{1}$ K. Nishikawa, ${ }^{13,}$ Y. Nishimura ${ }^{48}$ P. Novella, ${ }^{15}$ J. Nowak, ${ }^{25}$ H. M. O'Keeffe, ${ }^{25}$ R. Ohta, ${ }^{13,}$ K. Okumura, ${ }^{48,22}$ T. Okusawa, ${ }^{34}$ W. Oryszczak, ${ }^{53}$ S. M. Oser,,${ }^{3,51}$ T. Ovsyannikova, ${ }^{21}$ R. A. Owen, ${ }^{38}$ Y. Oyama, ${ }^{13,5}$ V. Palladino, ${ }^{18}$ J. L. Palomino, ${ }^{32}$ V. Paolone,${ }^{37}$ N. D. Patel, ${ }^{24}$ P. Paudyal, ${ }_{1}{ }^{26}$ M. Pavin, ${ }^{36}$ D. Payne, ${ }^{26}$ J. D. Perkin, ${ }^{43}$ Y. Petrov, ${ }^{3,51}$ L. Pickard, ${ }^{43}$ L. Pickering, ${ }^{16}$ E. S. Pinzon Guerra,${ }^{60}$ C. Pistillo, ${ }^{1}$ B. Popov ${ }^{36,}{ }^{* *}$ M. Posiadala-Zezula, ${ }^{53}$ J. -M. Poutissou, ${ }^{51}$ R. Poutissou, ${ }^{51}$ P. Przewlocki, ${ }^{31}$ B. Quilain, ${ }^{24}$ T. Radermacher ${ }^{42}$ E. Radicioni, ${ }^{17}$ P. N. Ratoff, ${ }^{25}$ M. Ravonel, ${ }^{11}$ M. A. Rayner, ${ }^{11}$ A. Redij, ${ }^{1}$ E. Reinherz-Aronis, ${ }^{7}$ C. Riccio ${ }^{18}$ P. Rojas, ${ }^{7}$ E. Rondio ${ }^{31}$ B. Rossi, ${ }^{18}$ S. Roth, ${ }^{42}$ A. Rubbia, ${ }^{10}$ A. C. Ruggeri, ${ }^{18}$ A. Rychter, ${ }^{54}$ R. Sacco, ${ }^{38}$ K. Sakashita, ${ }^{13, \$}$ F. Sánchez,${ }^{14}$ F. Sato, ${ }^{13}$ E. Scantamburlo, ${ }^{11}$ K. Scholberg, ${ }^{8,8}$ J. Schwehr, ${ }^{7}$ M. Scott, ${ }^{51}$ Y. Seiya ${ }^{34}$ T. Sekiguchi, ${ }^{13,}$ H. Sekiya, ${ }^{47,22, \|}$ D. Sgalaberna ${ }^{11}$ R. Shah, ${ }^{45,35}$ A. Shaikhiev ${ }^{21}$ F. Shaker ${ }^{57}$ D. Shaw, ${ }^{25}$ M. Shiozawa, ${ }^{47,22}$ T. Shirahige,${ }^{33}$ S. Short, ${ }^{38}$ M. Smy, ${ }^{4}$ J. T. Sobczyk, ${ }^{58}$ H. Sobel, ${ }^{4,22}$ M. Sorel, ${ }^{15}$ L. Southwell, ${ }^{25}$ P. Stamoulis, ${ }^{15}$ J. Steinmann, ${ }^{42}$ T. Stewart, ${ }^{45}$ P. Stowell, ${ }^{43}$ Y. Suda, ${ }^{46}$ S. Suvorov, ${ }^{21}$ A. Suzuki, ${ }^{23}$ K. Suzuki, ${ }^{24}$ S. Y. Suzuki, ${ }^{13, \$}$ Y. Suzuki ${ }^{22}$ R. Tacik, ${ }^{39,51}$ M. Tada, ${ }^{13, \$}$ S. Takahashi, ${ }^{24}$ A. Takeda, ${ }^{47}$ Y. Takeuchi, ${ }^{23,22}$ R. Tamura ${ }^{46}$ H. K. Tanaka ${ }^{47, \|}$ H. A. Tanaka, ${ }^{50,51, \dagger \dagger}$ D. Terhorst ${ }^{42}$ R. Terri, ${ }^{38}$ T. Thakore, ${ }^{27}$ L. F. Thompson, ${ }^{43}$ S. Tobayama, ${ }^{3,51}$ W. Toki, ${ }^{7}$ T. Tomura, ${ }^{47}$ C. Touramanis, ${ }^{26}$ T. Tsukamoto, ${ }^{13,}{ }^{3}$ M. Tzanov,${ }^{27}$ Y. Uchida, ${ }^{16}$ A. Vacheret, ${ }^{16}$ M. Vagins, ${ }^{22,4}$ Z. Vallari, ${ }^{32}$ G. Vasseur, ${ }^{5}$ C. Vilela, ${ }^{32}$ T. Vladisavljevic, ${ }^{35,22}$ T. Wachala, ${ }^{12}$ K. Wakamatsu, ${ }^{34}$ C. W. Walter, ${ }^{8,8}$ D. Wark, ${ }^{45,35}$ W. Warzycha, ${ }^{53}$ M. O. Wascko, ${ }^{16}$ A. Weber, ${ }^{45,35}$ R. Wendell, ${ }^{24, \|}$ R. J. Wilkes, ${ }^{56}$

M. J. Wilking, ${ }^{32}$ C. Wilkinson, ${ }^{1}$ J. R. Wilson, ${ }^{38}$ R. J. Wilson, ${ }^{7}$ C. Wret, ${ }^{16}$ Y. Yamada, ${ }^{13,4}$ K. Yamamoto, ${ }^{34}$ M. Yamamoto ${ }^{24}$ C. Yanagisawa, ${ }^{2,+*}$ T. Yano, ${ }^{23}$ S. Yen, ${ }^{51}$ N. Yershov, ${ }^{21}$ M. Yokoyama, ${ }^{46, \|}$ J. Yoo, ${ }^{27}$ K. Yoshida, ${ }^{24}$ T. Yuan, ${ }^{6}$ M. Yu, ${ }^{60}$ A. Zalewska, ${ }^{12}$ J. Zalipska, ${ }^{31}$ L. Zambelli, ${ }^{13, \ddagger}$ K. Zaremba, ${ }^{54}$ M. Ziembicki, ${ }^{54}$ E. D. Zimmerman, ${ }^{6}$ M. Zito, ${ }^{5}$ and J. Żmuda ${ }^{58}$

(T2K Collaboration)

\footnotetext{
${ }^{1}$ University of Bern, Albert Einstein Center for Fundamental Physics, Laboratory for High Energy Physics (LHEP), Bern, Switzerland ${ }^{2}$ Boston University, Department of Physics, Boston, Massachusetts, USA ${ }^{3}$ University of British Columbia, Department of Physics and Astronomy, Vancouver, British Columbia, Canada
} 
${ }^{4}$ University of California, Irvine, Department of Physics and Astronomy, Irvine, California, USA

${ }^{5}$ IRFU, CEA Saclay, Gif-sur-Yvette, France

${ }^{6}$ University of Colorado at Boulder, Department of Physics, Boulder, Colorado, USA

${ }^{7}$ Colorado State University, Department of Physics, Fort Collins, Colorado, USA

${ }^{8}$ Duke University, Department of Physics, Durham, North Carolina, USA

${ }^{9}$ Ecole Polytechnique, IN2P3-CNRS, Laboratoire Leprince-Ringuet, Palaiseau, France

${ }^{10}$ ETH Zurich, Institute for Particle Physics, Zurich, Switzerland

${ }^{11}$ University of Geneva, Section de Physique, DPNC, Geneva, Switzerland

${ }^{12}$ H. Niewodniczanski Institute of Nuclear Physics PAN, Cracow, Poland

${ }^{13}$ High Energy Accelerator Research Organization (KEK), Tsukuba, Ibaraki, Japan

${ }^{14}$ Institut de Fisica d'Altes Energies (IFAE), The Barcelona Institute of Science and Technology, Campus UAB, Bellaterra (Barcelona) Spain

${ }^{15}$ IFIC (CSIC \& University of Valencia), Valencia, Spain

${ }^{16}$ Imperial College London, Department of Physics, London, United Kingdom

${ }^{17}$ INFN Sezione di Bari and Università e Politecnico di Bari, Dipartimento Interuniversitario di Fisica, Bari, Italy

${ }^{18}$ INFN Sezione di Napoli and Università di Napoli, Dipartimento di Fisica, Napoli, Italy

${ }^{19}$ INFN Sezione di Padova and Università di Padova, Dipartimento di Fisica, Padova, Italy

${ }^{20}$ INFN Sezione di Roma and Università di Roma "La Sapienza", Roma, Italy

${ }^{21}$ Institute for Nuclear Research of the Russian Academy of Sciences, Moscow, Russia

${ }^{22}$ Kavli Institute for the Physics and Mathematics of the Universe (WPI),

The University of Tokyo Institutes for Advanced Study, University of Tokyo, Kashiwa, Chiba, Japan

${ }^{23}$ Kobe University, Kobe, Japan

${ }^{24}$ Kyoto University, Department of Physics, Kyoto, Japan

${ }^{25}$ Lancaster University, Physics Department, Lancaster, United Kingdom

${ }^{26}$ University of Liverpool, Department of Physics, Liverpool, United Kingdom

${ }^{27}$ Louisiana State University, Department of Physics and Astronomy, Baton Rouge, Louisiana, USA

${ }^{28}$ Université de Lyon, Université Claude Bernard Lyon 1, IPN Lyon (IN2P3), Villeurbanne, France

${ }^{29}$ Michigan State University, Department of Physics and Astronomy, East Lansing, Michigan, USA

${ }^{30}$ Miyagi University of Education, Department of Physics, Sendai, Japan

${ }^{31}$ National Centre for Nuclear Research, Warsaw, Poland

${ }^{32}$ State University of New York at Stony Brook,

Department of Physics and Astronomy, Stony Brook, New York, USA

${ }^{33}$ Okayama University, Department of Physics, Okayama, Japan

${ }^{34}$ Osaka City University, Department of Physics, Osaka, Japan

${ }^{35}$ Oxford University, Department of Physics, Oxford, United Kingdom

${ }^{36} U P M C$, Université Paris Diderot, CNRS/IN2P3,

Laboratoire de Physique Nucléaire et de Hautes Energies (LPNHE), Paris, France

${ }^{37}$ University of Pittsburgh, Department of Physics and Astronomy, Pittsburgh, Pennsylvania, USA

${ }^{38}$ Queen Mary University of London, School of Physics and Astronomy, London, United Kingdom

${ }^{39}$ University of Regina, Department of Physics, Regina, Saskatchewan, Canada

${ }^{40}$ University of Rochester, Department of Physics and Astronomy, Rochester, New York, USA

${ }^{41}$ Royal Holloway University of London, Department of Physics, Egham, Surrey, United Kingdom

${ }^{42}$ RWTH Aachen University, III. Physikalisches Institut, Aachen, Germany

${ }^{43}$ University of Sheffield, Department of Physics and Astronomy, Sheffield, United Kingdom

${ }^{44}$ University of Silesia, Institute of Physics, Katowice, Poland

${ }^{45}$ STFC, Rutherford Appleton Laboratory, Harwell Oxford, and Daresbury Laboratory,

Warrington, United Kingdom

${ }^{46}$ University of Tokyo, Department of Physics, Tokyo, Japan

${ }^{47}$ University of Tokyo, Institute for Cosmic Ray Research, Kamioka Observatory, Kamioka, Japan

${ }^{48}$ University of Tokyo, Institute for Cosmic Ray Research,

Research Center for Cosmic Neutrinos, Kashiwa, Japan

${ }^{49}$ Tokyo Metropolitan University, Department of Physics, Tokyo, Japan

${ }^{50}$ University of Toronto, Department of Physics, Toronto, Ontario, Canada

${ }^{51}$ TRIUMF, Vancouver, British Columbia, Canada

${ }^{52}$ University of Victoria, Department of Physics and Astronomy, Victoria, British Columbia, Canada

${ }^{53}$ University of Warsaw, Faculty of Physics, Warsaw, Poland

${ }^{54}$ Warsaw University of Technology, Institute of Radioelectronics, Warsaw, Poland

${ }^{55}$ University of Warwick, Department of Physics, Coventry, United Kingdom

${ }^{56}$ University of Washington, Department of Physics, Seattle, Washington, USA 


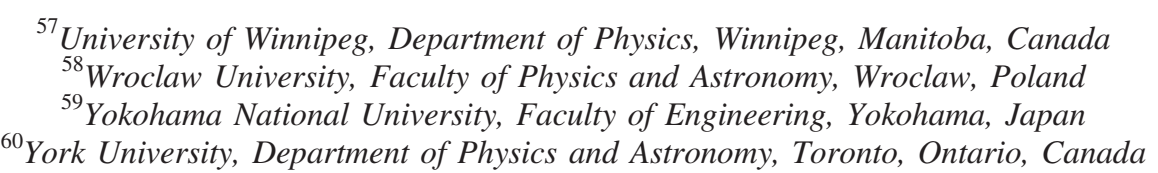

(Received 7 March 2017; published 29 June 2017)

\begin{abstract}
A class of extensions of the Standard Model allows Lorentz and CPT violations, which can be identified by the observation of sidereal modulations in the neutrino interaction rate. A search for such modulations was performed using the T2K on-axis near detector. Two complementary methods were used in this study, both of which resulted in no evidence of a signal. Limits on associated Lorentz and CPT-violating terms from the Standard Model extension have been derived by taking into account their correlations in this model for the first time. These results imply such symmetry violations are suppressed by a factor of more than $10^{20}$ at the $\mathrm{GeV}$ scale.
\end{abstract}

DOI: $10.1103 /$ PhysRevD.95.111101

\section{INTRODUCTION}

While Lorentz invariance is a cornerstone of the Standard Model (SM) of particle physics, violations of this symmetry are allowed in a variety of models [1-3] at or around the Planck scale, $m_{P} \sim 10^{19} \mathrm{GeV}$. At energies relevant to modern experiments, Lorentz invariance-violating (LV) processes are expected to be suppressed at least by $\sim 1 / m_{P}$. Experimental observations of such phenomena would provide direct access to physics at the Planck scale, and precision tests have been performed to overcome this suppression (cf. Ref. [4] for a review). Neutrino oscillations can be used as a natural interferometer to probe even weak departures from this symmetry and have been studied with accelerator [5-10], reactor [11], and atmospheric [12,13] neutrinos.

Lorentz and $C P T$ symmetry violations can be described within the context of the Standard Model extension (SME) [14], an observer-independent effective field theory that incorporates all possible spontaneous LV operators with the SM Lagrangian. In general, the SME allows two classes of effects for neutrino oscillations, sidereal violations, in which the presence of a preferred spatial direction induces oscillation effects that vary with the neutrino travel

*Deceased.

${ }^{\dagger}$ Now at CERN.

*Also at J-PARC, Tokai, Japan.

${ }^{\S}$ Affiliated member at Kavli IPMU (WPI), the University of Tokyo, Japan.

"Affiliated member at Kavli IPMU (WPI), the University of Tokyo, Japan.

"Also at National Research Nuclear University "MEPhI" and Moscow Institute of Physics and Technology, Moscow, Russia.

*Also at JINR, Dubna, Russia.

${ }^{\dagger}$ Also at Institute of Particle Physics, Canada.

\#Also at BMCC/CUNY, Science Department, New York, New York, USA.

Published by the American Physical Society under the terms of the Creative Commons Attribution 4.0 International license. Further distribution of this work must maintain attribution to the author(s) and the published article's title, journal citation, and DOI. direction, and spectral anomalies [15-17]. For a terrestrial fixed-baseline experiment, the rotation of the Earth induces a change in the direction of the neutrino target-detector vector relative to a fixed coordinate system such that a $\mathrm{LV}$ signal of the former type would manifest itself as a variation in the neutrino oscillation probability with sidereal time.

This paper reports a search for evidence of siderealdependent $\nu_{\mu}$ disappearance over an average baseline of $233.6 \mathrm{~m}$ using the T2K experiment. After introducing Lorentz invariance-violating oscillations within the SME and describing the $\mathrm{T} 2 \mathrm{~K}$ experiment, the selection of an analysis sample composed predominately of muon neutrinos inside the INGRID [18,19] detector is presented. Results of two complementary analyses of the data and concluding remarks follow thereafter.

\section{LV EFFECTS ON NEUTRINO OSCILLATIONS AT SHORT DISTANCES}

In this analysis, the LV is probed through $\nu_{\mu}$ disappearance channel. In the SME framework, the disappearance probability of a $\nu_{\mu}$ over short baselines is given by [16]

$$
\begin{aligned}
P_{\nu_{\mu} \rightarrow \nu_{\mu}}= & 1-\sum_{b, b \neq \mu} \frac{L^{2}}{(\hbar c)^{2}} \mid \mathcal{C}_{\mu b} \\
& +\left(\mathcal{A}_{s}\right)_{\mu b} \sin \left(\omega_{\oplus} T_{\oplus}\right)+\left(\mathcal{A}_{c}\right)_{\mu b} \cos \left(\omega_{\oplus} T_{\oplus}\right) \\
& +\left(\mathcal{B}_{s}\right)_{\mu b} \sin \left(2 \omega_{\oplus} T_{\oplus}\right)+\left.\left(\mathcal{B}_{c}\right)_{\mu b} \cos \left(2 \omega_{\oplus} T_{\oplus}\right)\right|^{2}
\end{aligned}
$$

where $L$ is the distance travelled before detection. Equation (1) is valid as long as $L \ll L_{\text {osc }}$, where $L_{\text {osc }}$ is the typical distance of standard $\nu_{\mu} \rightarrow \nu_{b}$ oscillations [20]. $T_{\oplus}$ is the local sidereal time, and $\omega_{\oplus}=\frac{2 \pi}{23^{h} 56^{m} 4.0916^{s}}$ is the Earth's sidereal frequency. Under a three-flavor neutrino hypothesis, oscillations of $\nu_{\mu}$ to $\nu_{e}$ and $\nu_{\tau}$ can occur. In general, the ten coefficients $\mathcal{C}_{\mu b},\left(\mathcal{A}_{c}\right)_{\mu b},\left(\mathcal{A}_{s}\right)_{\mu b},\left(\mathcal{B}_{c}\right)_{\mu b}$, and $\left(\mathcal{B}_{s}\right)_{\mu b}(b=e, \tau)$ are functions of the neutrino energy $E$, the neutrino beam direction at the time origin (see 
below), and of 40 parameters within the SME which carry explicit Lorentz and CPT-violation information: $\left(a_{L}\right)_{\mu b}^{\alpha}$ and $\left(c_{L}\right)_{\mu b}^{\alpha \beta}(b=e, \tau)[21]$. The $\left(a_{L}\right)_{\mu b}^{\alpha}\left(\left(c_{L}\right)_{\mu b}^{\alpha \beta}\right)$ are constant coefficients associated with $C P T$-odd (even) vector (tensor) fields. It should be noted that the impacts of $\left(a_{L}\right)_{a b}^{\alpha}$ and $\left(c_{L}\right)_{a b}^{\alpha \beta}$ on the set of ten coefficients depend on the absolute direction of the neutrino baseline [21]. In the analysis to follow, a search for sidereal variations is performed relative to an inertial frame centered on the Sun, assuming it to be stationary during the data taking period. Other than the choice of the origin of the time coordinate, this frame is the same as in Ref. [22]. The time origin $T=0$ is chosen as January 1, 1970, 09:00:00 Coordinated Universal Time. Data will be studied using the local sidereal phase (LSP), which is defined as LSP $=\bmod \left(T_{\oplus} \omega_{\oplus} / 2 \pi\right)$.

\section{EXPERIMENTAL SETUP}

The T2K long-baseline neutrino experiment uses the collision of $30 \mathrm{GeV}$ protons from the Japan Proton Accelerator Research Complex with a graphite target and focuses charged mesons produced in the subsequent interactions along the primary proton beam direction using a series of magnetic horns. Downstream of the production target is a 96-m-long decay volume in which these mesons decay to produce a beam of primarily muon neutrinos (99.3\% $\nu_{\mu}+\bar{\nu}_{\mu}$ along the beam axis).

This study is based on data accumulated from 2010 to 2013, divided into four run periods, and corresponds to $6.63 \times 10^{20}$ protons on target (POT) exposure of the INGRID detector in the neutrino mode. The neutrino beam is defined by the beam colatitude $\chi=53.55087^{\circ}$ in the Earth-centered frame with the same fixed axis as the Sun-centered frame. At the beamline location, a local frame is defined where the $\mathrm{z}$ axis corresponds to the zenith. The beam direction in this local frame is defined by the zenith angle $\theta=93.637^{\circ}$, and at the azimuthal angle, $\phi=270.319^{\circ}$. A more detailed description of the $\mathrm{T} 2 \mathrm{~K}$ experiment can be found in Ref. [18].

The INGRID detector is located $280 \mathrm{~m}$ downstream of the graphite target and is composed of $14120 \times 120 \times$ $109 \mathrm{~cm}$ modules assembled in a cross-shaped structure. Each module holds 11 tracking segments built from pairs of orthogonally oriented scintillator planes interleaved with nine iron planes. The scintillator planes are built from 24 plastic scintillator bars connected to multipixel photon counters (MPPCs). Situated on the beam center, INGRID's high event rate makes it well suited to a search for a sidereal variation in the $\nu_{\mu}$ interactions.

Although the $\nu_{\mu} \rightarrow \nu_{\mu}$ oscillation probability in Eq. (1) depends on the square of the neutrino flight length, the precise distance from creation to detection for each neutrino is unknown. Indeed, the neutrino's parent meson may decay anywhere along the decay volume as shown in Fig. 1. As a result, the present analysis uses the mean of this distribution,

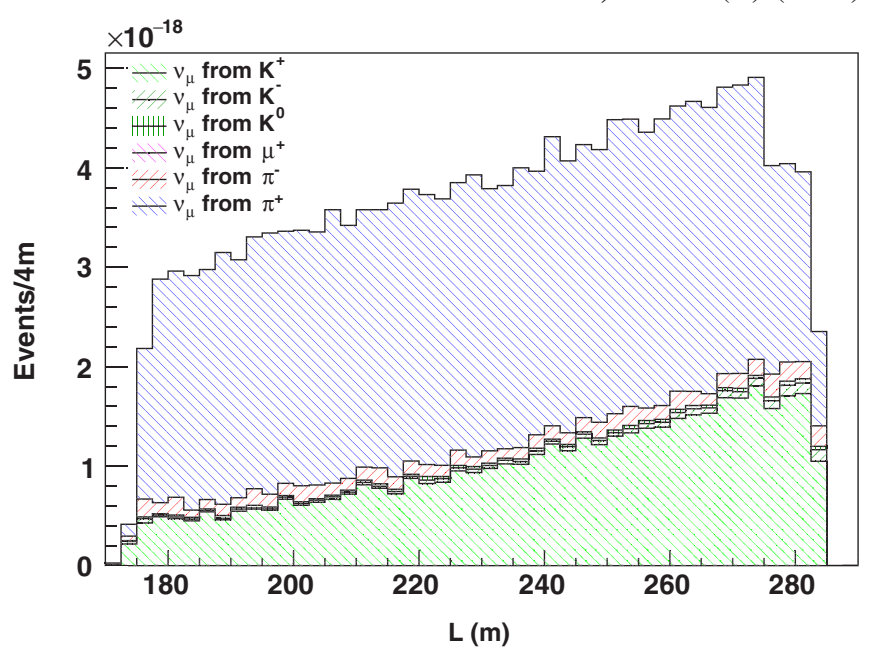

FIG. 1. Flight length to the INGRID detector for MC $\nu_{\mu}$ produced in the T2K decay volume. The distribution is separated based on the neutrino's parent particle.

$L_{\text {ave }}=233.6 \mathrm{~m}$, as an effective distance travelled for all candidate events. Similarly, the mean neutrino energy of the flux at the INGRID detector, $E_{\text {ave }}=2.7 \mathrm{GeV}$, is used.

\section{IV. $\nu_{\mu}$ EVENT SELECTION AND SYSTEMATIC UNCERTAINTIES}

\section{A. INGRID $\nu_{\mu}$ event selection}

To prevent LV oscillation-induced $\nu_{e}$ and $\nu_{\tau}$ from washing out an LV effect on the $\nu_{\mu}$ data, it is essential to select a sample with very high $\nu_{\mu}$ purity. Since the $\nu_{\tau}$ charged-current (CC) interactions have a $3.5 \mathrm{GeV}$ production threshold, their cross section in the $\mathrm{T} 2 \mathrm{~K}$ energy range is very small. Their impact on the analysis was evaluated to be negligible. Consequently, no attempts were made to further reject them in the signal selection. Charged-current neutrino $\nu_{\mu}$ interactions within INGRID are identified by a reconstructed track consistent with a muon originating in the detector fiducial volume and coincident in time with the expected arrival of neutrinos in the beam originated from a given proton bunch. In addition to a set of cuts to define a basic leptonlike sample [23], a likelihood function, hereafter referred to as muon confidence level $\left(\mu_{\mathrm{CL}}\right)$, is used to further separate tracks produced by muons from showers produced by electrons or hadrons. This function is based on four discriminating variables: the number of active scintillator bars transverse to the beam direction averaged over the number of active planes, i.e., planes having at least one hit belonging to the track; the primary track's length; the dispersion of the track's energy deposition with distance; and the number of active scintillator bars close to the primary interaction vertex. The first three variables focus on the tendency for showers to have a broader transverse development and varying rate of energy deposition, whereas muons at $\mathrm{T} 2 \mathrm{~K}$ energies are minimum ionizing 


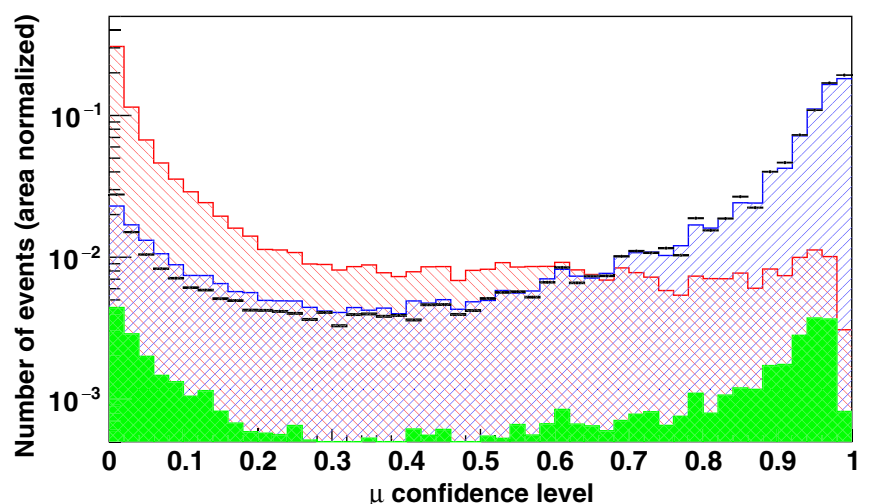

FIG. 2. Distribution of the $\mu_{\mathrm{CL}}$ variable for $\nu_{\mu} \mathrm{CC}$ (blue), $\nu_{e} \mathrm{CC}$ (red), and NC events (green) from the MC are overlaid with data (black). The data, $\nu_{\mu} \mathrm{CC}$, and $\nu_{\mu} \mathrm{NC}$ histograms are first normalized by protons on target and then scaled by one over the number of $\nu_{\mu} \mathrm{CC}$ events to preserve their relative proportions. The $\nu_{e}$ CC histogram is area normalized to compare with the $\nu_{\mu}$ $\mathrm{CC}$ histogram. The pink arrow represents the lower cut value on the $\mu_{\mathrm{CL}}$ that defines the $\nu_{\mu}$ event selection.

and are more longitudinally penetrating. The fourth variable is based on a region defined by only the two planes upstream and downstream of the event vertex and is useful for discriminating against showers with additional particles near the event vertex and proton-induced activity. Since the total neutrino flux is constant and the neutral current (NC) cross section is the same for each neutrino flavor, the NC event rate within INGRID is expected to be constant with sidereal time. Accordingly, no additional cuts to remove $\mathrm{NC}$ events are used. Figure 2 shows the $\mu_{\mathrm{CL}}$ likelihood distribution for reconstructed data and Monte Carlo (MC) $\nu_{\mu} \mathrm{CC}, \nu_{e} \mathrm{CC}$, and NC interactions. A cut on $\mu_{\mathrm{CL}} \geq 0.54$ has been selected to ensure that the $\nu_{e}$ contamination of the final sample is smaller than the statistical error on the $\nu_{\mu}$ component while maximizing the $\nu_{\mu}$ statistics. After applying all analysis cuts, the $\nu_{\mu} \mathrm{CC}$ selection efficiency is $\epsilon_{\mu}=44.0 \%$. The corresponding $\nu_{e}$ efficiency, $\epsilon_{e}$, has been reduced to $13.3 \%$. There are $6.75 \times 10^{6}$ events remaining in the final sample, which provides an average statistical error of $0.22 \%$ in each of the 32 analysis bins (defined below). If an oscillation effect equivalent to three times the statistical error on the $\nu_{\mu}$ component appears as $\nu_{e}$ in the final sample, the resulting contamination will be $0.2 \%$. Assuming no oscillation due to LV effect, the final sample has $3.4 \% \mathrm{NC}$ events.

\section{B. Timing corrections and systematic uncertainties}

The operation of the T2K beam is not constant in time and varies with the hour of the day and season of the year. The effect of time-dependent changes in the neutrino event rate must be corrected since they can mimic an LVoscillation signal or reduce the analysis sensitivity. Such effects can be separated into two distinct classes depending on whether they alter the neutrino beam itself or the INGRID detector. The first class consists of three timedependent corrections considered for the neutrino beam:

(i) Beam center variations during each run.-Since the neutrino interaction rate itself is insufficient to estimate these variations, muons collected spill by spill with a muon detector just downstream of the decay volume [24] are used to estimate the beam center position. For each of the four run periods considered in this exposure, the beam center position as a function of LSP is estimated after correcting for tidal effects at the detector. These data are then used to extrapolate the position of the neutrino beam center, which is aligned with the muon direction, at INGRID. LSP-dependent corrections to the observed event rate at INGRID due to shifts in the neutrino beam center are estimated using MC.

(ii) Beam center variation between runs.-Changes in the average beam center position between run periods are evaluated using the INGRID neutrino data, and a correction is estimated and applied as in the above.

(iii) Beam intensity variation between runs and nonuniform POT exposure as a function of LSP.-A correction is applied to bring the event rate per POT in each LSP bin in line with the average for the entire run. The correction is applied for each event based on its run and sidereal phase. A further correction is applied to make the average event rate per POT of each run consistent with that of a reference run chosen to be near the end of the data taking period.

The second class of effects consists of three additional corrections to account for changes in the response of INGRID:

(i) Event pileup variations.-Typically, only single interactions in an INGRID module are reconstructed, and other interactions in the same data acquisition timing window (one for each neutrino bunch) are lost (pileup events). However, changes in the beam intensity affect the probability of multiple interactions within an INGRID reconstruction timing window. Accordingly, events at INGRID are corrected as a linear function of LSP to account for the variation in pileup events with variations in the beam intensity. The number of lost pileup events varies between 3\% and 7\% across the INGRID modules.

(ii) Dark noise variations. - Variations in the temperature and humidity affect the MPPC dark rate, which in turn weakly affects the neutrino detection efficiency. The maximal variations of the dark rate with the sidereal time is $2 \%$. A correction to account for this efficiency variation has been applied linearly with the dark rate. 


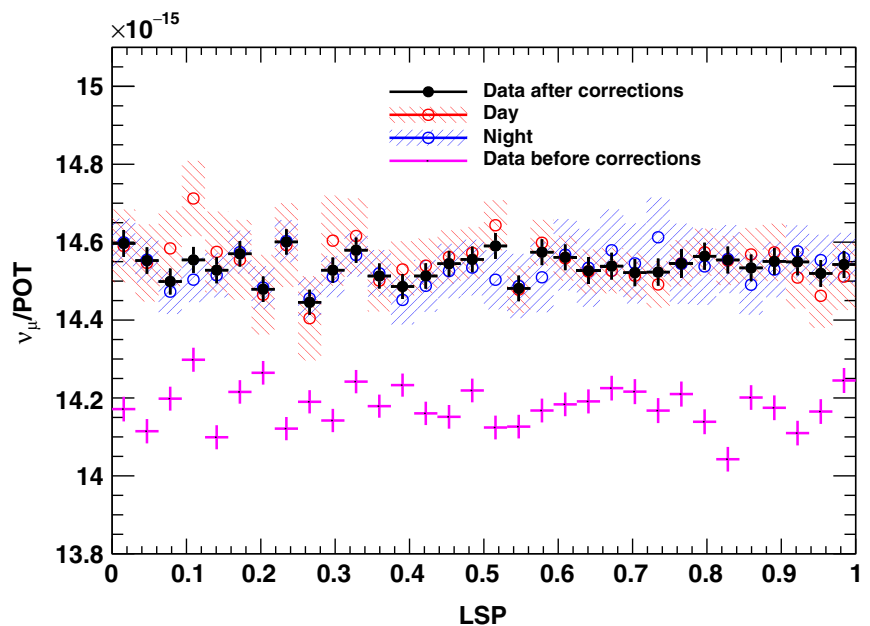

FIG. 3. Distribution of reconstructed $\mu$-like events per POT as a function of LSP. Data before (magenta) and after (black) corrections are shown together with the corrected sample additionally split into day (red) and night (blue) subsamples.

(iii) Variations in the photosensor gain.-The MPPC gain is influenced by environmental changes, and the scintillator gain might decrease over time. Gain changes impact both the reconstruction and the analysis selection and are corrected using a sample of beam-induced muon interactions in the rock upstream of INGRID. The effect of variations in the charge at the minimum ionization peak of these muons is simulated in $\mathrm{MC}$ and used to correct the neutrino event rate. The size of the correction varies with LSP and does not exceed $1 \%$.

The validity of the above corrections has been tested by separating the analysis data set into day and night subsamples. Though time-dependent differences are expected in the split samples due to, for instance, cooler temperatures at night or beamline maintenance during the day, the data should be consistent with one another when viewed in the LSP coordinate if the above corrections have been applied consistently. Figure 3 shows the day and night distributions as a function of LSP. The agreement between the day and night distribution is evaluated with a Pearsons chi-squared

TABLE I. Summary of the $1 \sigma$ systematic uncertainties induced from correcting for time-dependent variations in the neutrino event rate. The beam position variation between and within run periods has been combined into a single entry in the table.

\begin{tabular}{lc}
\hline \hline Source & Systematic uncertainty $(\%)$ \\
\hline Pileup & 0.01 \\
MPPC dark noise & 0.01 \\
MPPC gain variation & 0.06 \\
Beam position & 0.03 \\
Beam intensity & 0.05 \\
Total systematic & 0.08 \\
\hline \hline
\end{tabular}

test, and a corresponding $\chi^{2} / \mathrm{NDF}=28.3 / 32$ has been found. Data before and after all corrections also appear in the figure. Systematic errors for each of the corrections have been evaluated and are listed in Table I. The total systematic error is $0.08 \%$, which is small when compared to the statistical error of the final sample, $0.22 \%$.

\section{ANALYSIS METHODOLOGY AND RESULTS}

The analysis of the final data sample is performed in two stages. First, the compatibility of the data with a null signal is studied using a fast Fourier transform (FFT) method (Sec. VA). This method explicitly searches for a sidereal modulation and ultimately provides an estimate of the power of each Fourier mode from a potential signal. Then, constraints on the parameters appearing in Eq. (1) are extracted using a likelihood method (Sec. V B) that includes their correlations. Figure 4 shows examples of the expected LSP distribution for MC generated under three signal assumptions.

\section{A. Fast Fourier transform result}

Expanding Eq. (1) indicates that LV oscillations are described by four harmonic sidereal frequencies, $f_{i}=i \cdot \omega_{\oplus}$, $i \in[1,4]$, and a constant term. The FFT [25,26] method is most efficient for $N=2^{L}$ bins, and the sensitivity of the current analysis is found to be optimal when $L=5$. Data are therefore divided into 32 evenly spaced LSP bins for input into the FFT, and the magnitudes of the four Fourier modes, $\left|F_{i}\right|$, are then estimated. Note that the constant term is not considered in this study due to large uncertainties in the beam flux normalization. A $3 \sigma$ detection threshold has

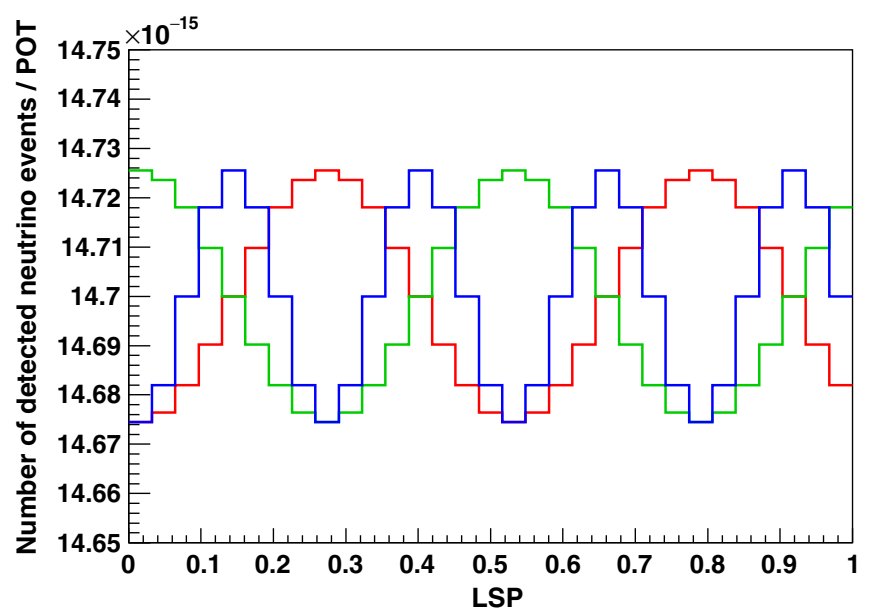

FIG. 4. Distribution of the $\nu_{\mu}$ event rate as a function of LSP for three different assumed signal configurations: $\left(\mathcal{C}_{\mu e},\left(\mathcal{A}_{c}\right)_{\mu e},\left(\mathcal{A}_{s}\right)_{\mu e},\left(\mathcal{B}_{c}\right)_{\mu e},\left(\mathcal{B}_{s}\right)_{\mu e}\right)=\left(0,5 \times 10^{-20}, 0,0,0\right) \mathrm{GeV}$ (red), $\quad\left(0,0,5 \times 10^{-20}, 0,0\right) \mathrm{GeV}$ (green), and $(0,0,0,5 \times$ $\left.10^{-20}, 0\right) \mathrm{GeV}$ (blue). The coefficients corresponding to $\nu_{\mu} \rightarrow \nu_{\tau}$ oscillation $\left(\mathcal{C}_{\mu \tau},\left(\mathcal{A}_{c}\right)_{\mu \tau},\left(\mathcal{A}_{s}\right)_{\mu \tau},\left(\mathcal{B}_{c}\right)_{\mu \tau},\left(\mathcal{B}_{s}\right)_{\mu \tau}\right)$ have been set to zero. 
TABLE II. Observed power in each Fourier mode from a fit to the data using the FFT method. A positive observation at $3 \sigma$ would correspond to an observed power greater than 0.026 in any $\omega_{\oplus}$.

\begin{tabular}{lcc}
\hline \hline Fourier mode & Magnitude & $\mathrm{p}$ value \\
\hline$\left|F_{1}\right|$ & 0.011 & 0.35 \\
$\left|F_{2}\right|$ & 0.009 & 0.48 \\
$\left|F_{3}\right|$ & 0.006 & 0.69 \\
$\left|F_{4}\right|$ & 0.009 & 0.51 \\
\hline \hline
\end{tabular}

been determined as the power in a Fourier mode for which $0.3 \%$ of $\mathrm{MC}$ experiments generated without $\mathrm{LV}$ effects shows higher power. For each mode, this threshold corresponds to $\left|F_{i}\right|>0.026$. The results of the fit to the data are shown in Table II together with a $\mathrm{p}$ value estimating the likelihood that the observed power was produced by a statistical fluctuation of the null (no LV) hypothesis. All $\left|F_{i}\right|$ are below the $3 \sigma$ detection threshold and indicate no evidence for a LV signal.

Constraints on the SME coefficients can be extracted with the FFT method $[7,21]$ under the assumption that the parameters above are uncorrelated. However, since the data sets are reduced to the four amplitudes and the relatively large number of parameters in the oscillation function, correlations are expected. Figure 5 shows the probability for data without LV to yield more power in the Fourier modes than the average expected for a LV signal as a function of the SME coefficients $\left(a_{L}\right)_{\mu e}^{X}$ and $\left(c_{L}\right)_{\mu e}^{T X}$. The parameters exhibit a high degree of anticorrelation, indicating that in the event of a null observation as above using the FFT method without considering these correlations may lead to an underestimation of the parameter limits. As the parameters in Eq. (1) are functions of these coefficients, they might be also expected to exhibit correlations. Accordingly, a likelihood method has been developed to

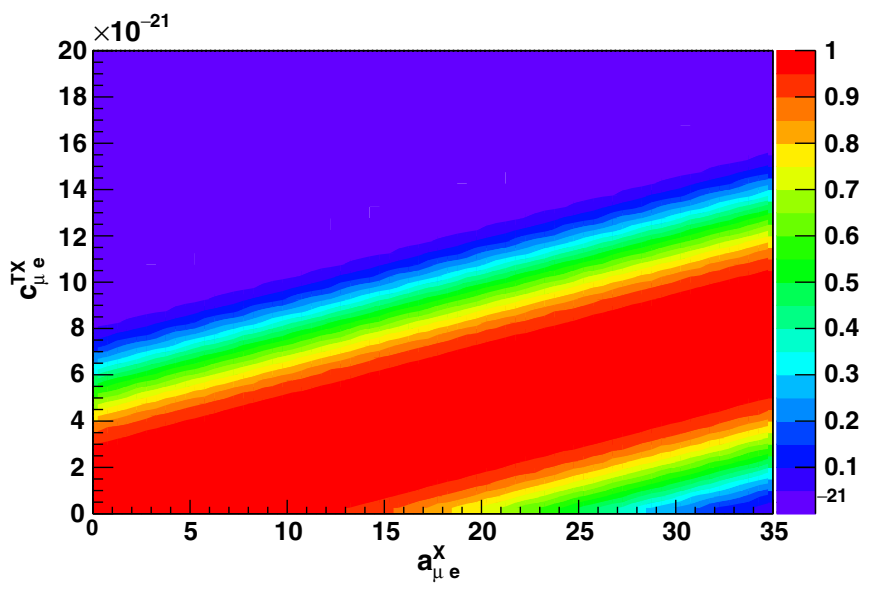

FIG. 5. Probability for the observed Fourier power in a null observation to exceed the expected power from a LV signal as a function of the $\left(a_{L}\right)_{\mu e}^{X}$ and $\left(c_{L}\right)_{\mu e}^{T X}$ coefficients. fully incorporate these correlations when making parameter estimations.

\section{B. Likelihood analysis}

Because of the large number of SME parameters [21] relative to the number of observables, this analysis does not estimate the $\left(a_{L}\right)_{a b}^{X}$ and $\left(c_{L}\right)_{a b}^{T X}$ parameters but the $\mathcal{C}_{\mu b}$, $\left(\mathcal{A}_{c}\right)_{\mu b},\left(\mathcal{A}_{s}\right)_{\mu b},\left(\mathcal{B}_{c}\right)_{\mu b},\left(\mathcal{B}_{s}\right)_{\mu b}(b=e, \tau)$ parameters from Eq. (1) using a likelihood method that fully incorporates their correlations and the experimental uncertainties. However, since the impact of systematic errors is negligible (cf. Table I), only the statistical uncertainty in each LSP bin is considered here. Further, each parameter is assumed to be real valued. Sensitivity studies without this assumption showed no significant constraint on the complex phases of these parameters with the present data. Under these conditions, a simultaneous fit for ten real parameters using the data and binning from the previous section has been performed. Since the parameters are highly correlated, the contours and limits are not estimated assuming a profiling method but instead using a likelihood marginalization which genuinely preserves their correlations [27]. This analysis assumes flat priors for all the parameters since no LV has been discovered so far. The results of the fit are shown in Table III.

As expected from the FFT method, no indications of LV oscillations are found, and $2 \sigma$ upper limits are set for each parameter. Those limits are compared with the sensitivity obtained by determining the parameter absolute values for which 5\% of some MC experiments generated without LV effects shows higher absolute values. The contour limits are constructed following a constant $\Delta \chi^{2}$ method and are shown in Fig. 6 for the $\left(\mathcal{A}_{c}\right)_{\mu e}$ and $\left(\mathcal{A}_{s}\right)_{\mu e}$ parameters that show important anticorrelations. While correlatedparameter analyses have been performed elsewhere [22], this is the first search to do so using all ten parameters simultaneously. The five harmonics in Eq. (1) heavily correlate the ten parameters as shown in Fig. 6.

TABLE III. Best fit values with $68 \%$ and $95 \%$ upper limit values on the LV model parameters using the likelihood method (in units of $10^{-20} \mathrm{GeV}$ ). In the last row, the expected sensitivity is shown.

\begin{tabular}{lrcccc}
\hline \hline & $\mathcal{C}_{\mu e}$ & $\left(\mathcal{A}_{c}\right)_{\mu e}$ & $\left(\mathcal{A}_{s}\right)_{\mu e}$ & $\left(\mathcal{B}_{c}\right)_{\mu e}$ & $\left(\mathcal{B}_{s}\right)_{\mu e}$ \\
\hline Best fits & -0.3 & 0.3 & 0.4 & -1.2 & 2.0 \\
68\% C.L. & 1.3 & 1.5 & 2.0 & 1.3 & 1.6 \\
95\% C.L. & 3.0 & 3.2 & 3.8 & 2.6 & 3.1 \\
95\% C.L. sensitivity & 2.5 & 2.7 & 4.3 & 3.5 & 3.5 \\
& $\mathcal{C}_{\mu \tau}$ & $\left(\mathcal{A}_{c}\right)_{\mu \tau}$ & $\left(\mathcal{A}_{s}\right)_{\mu \tau}$ & $\left(\mathcal{B}_{c}\right)_{\mu \tau}$ & $\left(\mathcal{B}_{s}\right)_{\mu \tau}$ \\
Best fits & -0.8 & -0.4 & -3.2 & -0.4 & 1.1 \\
68\% C.L. & 1.3 & 1.5 & 2.0 & 1.3 & 1.6 \\
95\% C.L. & 3.0 & 3.2 & 3.8 & 2.6 & 3.1 \\
95\% C.L. sensitivity & 2.5 & 2.7 & 4.3 & 3.5 & 3.5 \\
\hline \hline
\end{tabular}




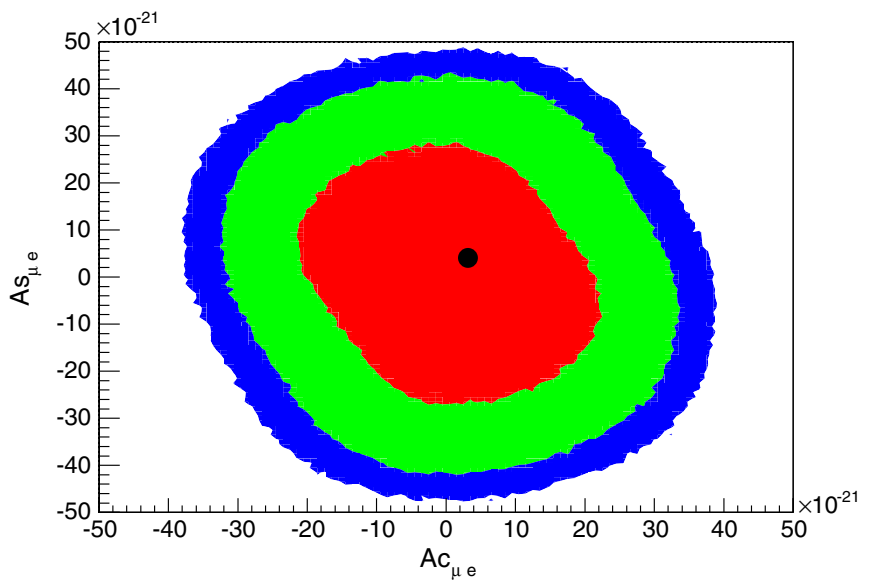

FIG. 6. Ten-coefficient fit result in the $\left(\mathcal{A}_{c}\right)_{\mu e},\left(\mathcal{A}_{s}\right)_{\mu e}$ coefficient plane. The other parameters are marginalized over. The bestfit point is marked in black, with $68 \%, 90 \%$, and $95 \%$ credible intervals shown in red, green and blue, respectively.

Neglecting the correlations between the parameters will lead an underestimation of the parameter limits. Since these correlations vary with the direction and position of each experiment, any comparison or combination of the limits found by different experiments requires preserving these correlations.

\section{CONCLUSIONS}

The T2K experiment has performed a search for Lorentz and $C P T$-invariance violations using the INGRID on-axis near detector. Two complementary analysis methods have found no evidence of such symmetry violations for the energy, neutrino baseline, and data set used [28]. Not only are the data consistent with an LSP-independent event rate based on a FFT analysis, but a likelihood analysis incorporating parameter correlations has corroborated this finding and yielded constraints on ten SME parameters.

\section{ACKNOWLEDGMENTS}

We thank the J-PARC staff for superb accelerator performance. We thank the CERN NA61 Collaboration for providing valuable particle production data. We acknowledge the support of MEXT, Japan; NSERC (Grant No. SAPPJ-2014-00031), NRC and CFI, Canada; CEA and CNRS/IN2P3, France; DFG, Germany; INFN, Italy; National Science Centre (NCN) and Ministry of Science and Higher Education, Poland; RSF, RFBR, and MES, Russia; MINECO and ERDF funds, Spain; SNSF and SERI, Switzerland; STFC, UK; and DOE, USA. We also thank CERN for the UA1/NOMAD magnet, DESY for the HERA-B magnet mover system, NII for SINET4, the WestGrid and SciNet consortia in Compute Canada, and GridPP in the United Kingdom. In addition, participation of individual researchers and institutions has been further supported by funds from ERC (FP7), H2020 Grant No. RISE-GA644294-JENNIFER, EU; JSPS, Japan; Royal Society, UK; and the DOE Early Career Program, USA.
[1] V. A. Kostelecký and S. Samuel, Phys. Rev. D 39, 683 (1989).

[2] S. W. Hawking, Phys. Rev. D 14, 2460 (1976).

[3] I. Hinchliffe, N. Kersting, and Y. Ma, Int. J. Mod. Phys. A 19, 179 (2004).

[4] V. A. Kostelecký and N. Russell, Rev. Mod. Phys. 83, 11 (2011).

[5] L. B. Auerbach et al. (LSND Collaboration), Phys. Rev. D 72, 076004 (2005).

[6] A. A. Aguilar-Arevalo et al. (MiniBooNE Collaboration), Phys. Lett. B 718, 1303 (2013).

[7] P. Adamson et al. (MINOS Collaboration), Phys. Rev. Lett. 101, 151601 (2008).

[8] P. Adamson et al. (MINOS Collaboration), Phys. Rev. D 85, 031101 (2012).

[9] P. Adamson et al. (MINOS Collaboration), Phys. Rev. Lett. 105, 151601 (2010).

[10] B. Rebel and S. Mufson, Astropart. Phys. 48, 78 (2013).

[11] Y. Abe et al. (Double Chooz Collaboration), Phys. Rev. D 86, 112009 (2012).
[12] R. Abbasi et al. (IceCube Collaboration), Phys. Rev. D 82, 112003 (2010).

[13] K. Abe et al. (Super-Kamiokande Collaboration), Phys. Rev. D 91, 052003 (2015).

[14] D. Colladay and V. A. Kostelecký, Phys. Rev. D 58, 116002 (1998).

[15] V. A. Kostelecký and M. Mewes, Phys. Rev. D 85, 096005 (2012).

[16] V. A. Kostelecký and M. Mewes, Phys. Rev. D 70, 031902 (2004).

[17] V. A. Kostelecký and M. Mewes, Phys. Rev. D 69, 016005 (2004).

[18] K. Abe et al. (T2K Collaboration), Nucl. Instrum. Methods Phys. Res., Sect. A 659, 106 (2011).

[19] K. Abe et al. (T2K Collaboration), Nucl. Instrum. Methods Phys. Res., Sect. A 694, 211 (2012).

[20] K. A. Olive et al., Chin. Phys. C 38, 090001 (2014).

[21] J. S. Diaz, V. A. Kostelecký, and M. Mewes, Phys. Rev. D 80, 076007 (2009).

[22] T. Katori (MiniBooNE Collaboration), Mod. Phys. Lett. A 27, 1230024 (2012). 
[23] K. Abe et al. (T2K Collaboration), Phys. Rev. D 90, 052010 (2014).

[24] K. Abe et al. (T2K Collaboration), Prog. Theor. Exp. Phys. 5, 053C02 (2015).

[25] W. H. Press, Numerical Recipes: The Art of Scientific Computing, 3rd ed. (Cambridge University Press, Cambridge, England, 2007).
[26] P. Duhamel and V. Martin, Signal Processing 19, 259 (1990).

[27] C. Patrignani et al. (Particle Data Group Collaboration), Chin. Phys. C 40, 100001 (2016).

[28] K. Abe et al. (T2K Collaboration), T2K public data, http:// t2k-experiment.org/results/Ingrid-leptonviolation-2017. 